\title{
Tâches, possibilités et limites de la prise en charge des patients en fin de vie
}

\author{
M. Zimmermann-Acklin
}

Correspondance:

Markus Zimmermann-Acklin Institut für Sozialethik (ISE)

Université Lucerne

Gibraltarstrasse 3

Case postale 7763

CH-6000 Lucerne 7
La prise en charge des patients mourants représente souvent un défi exigeant pour les médecins et les soignants. Les espoirs des patients et de leurs proches, leurs attentes et leurs différentes conceptions d'une fin de vie convenable, les débats politiques avec leurs revendications juridiques et sociales contradictoires et, finalement, le développement constant des possibilités de traitement tant du point de vue médical que du point de vue technique exigent une réflexion constante sur la portée de ces défis, en particulier sur le plan de l'éthique. La discussion et la publication des dernières directives médicoéthiques pour la prise en charge des patients mourants remonte déjà à quelques années. C'est pourquoi, l'ASSM a décidé de réviser fondamentalement les «Directives médico-éthiques sur l'accompagnement médical des patients en fin de vie ou souffrant de troubles cérébraux extrêmes» datant de 1995 et de traiter les deux domaines dans des directives séparées. Alors que les directives pour «le traitement et la prise en charge des patients souffrant d'atteintes cérébrales extrêmes de longue durée» seront publiées prochainement dans leur version définitive, l'ASSM met en consultation les nouvelles directives pour «la prise en charge des patients en fin de vie».

Ces directives ont été élaborées sur une durée d'un an et demi par une sous-commission interdisciplinaire constituée par deux représentants des professions de soins, sept experts des différentes disciplines médicales et un représentant pour chacun des domaines suivants: le droit, la psychologie, l'assistance spirituelle et l'éthique. La sous-commission s'est réunie 12 fois (dont une retraite de deux jours); au cours de ces rencontres, elle a invité de nombreux experts externes. De plus, elle a soumis le texte à des représentant(e)s de l'ASI, de la FMH, des spécialistes de la médecine palliative, du droit et de la médecine générale dans le cadre d'une pré-consultation. Enfin, ces directives ont été discutées intensivement et réexaminées par les membres de la CCE, du Sénat et du comité de direction.

La définition des tâches, possibilités et limites de la prise en charge des patients en fin de vie a été au centre des discussions. A cet égard, et dans le sens de la prise en charge palliative, l'objectif primordial de toutes les mesures consiste à soulager les souffrances et à offrir au patient la meilleure qualité de vie possible ainsi qu'à soutenir ses proches.

Tout en restant claires quant à leur objectif principal, ces directives règlent toute une série de questions délicates, voire même controversées. On retiendra en particulier:

- La définition du champ d'application: comment peut-on distinguer clairement la situation d'un patient mourant de celle d'un patient gravement malade dont la prise en charge peut, le cas échéant, exiger d'autres priorités?

- La prise de décision pour les patients incapables de discernement: comment peut-on prendre des décisions si la volonté présumée du patient n'est que supposée, voire même méconnue?

- La mise en pratique de la prise en charge palliative: qu'englobe-t-elle exactement et comment peut-elle être mise en pratique?

- L'évaluation de l'euthanasie indirecte: Quel est le comportement adéquat à adopter depuis le soulagement des douleurs jusqu'à la possibilité de la sédation palliative?

- La réglementation de l'assistance au suicide: jusqu'où un médecin peut-il aller dans le cadre individuel, où sont les limites?

- La mise à disposition des ressources nécessaires: les ressources nécessaires sont-elles disponibles de facto pour être en mesure d'appliquer les principes énoncés dans ces directives?

Les réponses à ces questions complexes ont précisé des points importants en définissant clairement le champ d'application qui n'englobe pas les situations des patients souffrant d'atteintes extrêmes en dehors de la phase de fin de vie ainsi qu'en soulignant l'importance de l'autodétermination du patient et d'une prise en charge palliative complète et introduite en temps utile. De plus, la prise en compte de l'autodétermination du patient a finalement aussi contribué à une certaine ouverture quant à l'appréciation de l'assistance médicale au suicide: D'une part, l'assistance au suicide ne fait pas partie de l'activité médicale, le médecin étant tenu d'utiliser ses 
compétences médicales dans le but de soigner, soulager et accompagner son patient. D'autre part, il doit tenir compte de la volonté du patient, ce qui peut signifier que la décision morale et personnelle du médecin d'apporter une aide au suicide à un patient mourant, dans certains cas particuliers, doit être respectée. A chaque médecin incombe alors la responsabilité de vérifier si les exigences minimales suivantes sont réunies - ce qui est loin d'être toujours le cas dans la pratique actuelle -: notamment, si la fin de vie est proche, si des alternatives de traitements ont été proposées et, si souhaitées par le patient, mises en ouvre et si son désir de mourir est mûrement réfléchi et ne résulte pas d'une pression extérieure.
Le travail de la sous-commission a été empreint de la volonté de tous les participants de comprendre les opinions parfois très divergentes et de mettre en consultation des directives qui, en dépit des estimations différentes, donnent une orientation claire et mettent en place des garde-fous. On constatera à certaines formulations que la commission ne s'est pas facilitée le travail et a largement débattu et argumenté autour de nombreuses affirmations. Dans le cadre de la présente procédure de consultation, le corps médical est invité à soumettre ses remarques et suggestions au secrétariat général de l'ASSM (Petersplatz 13, 4051 Bâle ou e-mail: mail@samw.ch) 